Клиническая онкогематология. 2019;12(2):211-9

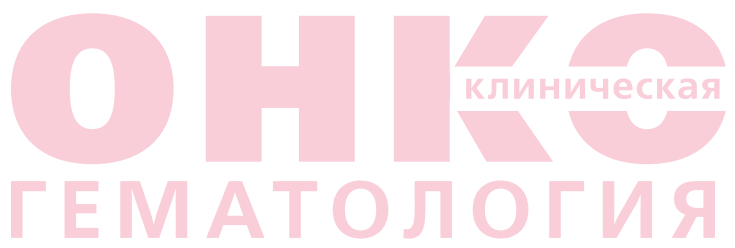

МИЕЛОИДНЫЕ ОПУХОЛИ

\section{Prognostic Value of Genetic Mutations in Patients with Acute Myeloid Leukemias: Results of a Cooperative Study of Hematology Clinics of Saint Petersburg (Russia) and Charite Clinic (Germany)}

\author{
Е.В. Мотыко', О.В. Блау², Л.Б. Полушкина', \\ Л.С. Мартыненко', М.П. Бакай', Н.Ю. Цыбакова', \\ Ю.С. Руженкова', Е.В. Клеина', Н.Б. Павленко', \\ А.М. Раджабова ${ }^{1}$, Е.В. Карягина ${ }^{3}$, О.С. Успенская ${ }^{4}$, \\ С.В. Волошин ${ }^{1}$, А.В. Чечеткин ${ }^{1}$, И.С. Мартынкевич
}

${ }^{1}$ ФГБУ «Российский НИИ гематологии и трансфузиологии ФМБА», 2-я
Советская ул., д. 16, Санкт-Петербург, Российская Федерация, 191024
${ }^{2}$ Клиника Шарите, Берлинский медицинский университет,
ул. Хинденбургдамм, д. 30, Берлин, Германия, 12200
${ }^{3}$ ГБУз «Городская больница № 15», ул. Авангардная, д. 4,
Санкт-Петербург, Российская Федерация, 198205
${ }^{4}$ ГБУз «Ленинградская областная клиническая больница», пр-т Луна-
чарского, д. 45-49, Санкт-Петербург, Российская Федерация, 194291

\section{РЕФ ЕРАТ}

Цель. Проанализировать влияние на прогноз ряда типичных для больных острыми миелоидными лейкозами (ОМЛ) мутаций.

Материалы и методы. В исследование включено 620 пациентов с ОМЛ, проходивших обследование в гематологических клиниках Санкт-Петербурга (Россия) и в клинике Шарите (Берлин, Германия). Цитогенетическое исследование выполнено методом G-дифференциального окрашивания хромосом. Скрининг аберраций в генах DNMT3A, IDH1/2 проводили с помощью полимеразной цепной реакции (ПЦР) в режиме реального времени с дальнейшим анализом кривых плавления и секвенирования. Мутации в генах FLT3, NPM1 определяли методом ПЦР.

Результаты. Мутации обнаружены у 343 (55,3 \%) из 620 больных. Статистически значимо чаще мутации определялись у больных с нормальным кариотипом (HK) ( $p=0,001)$. Наличие мутации FLT3-ITD было связано с уменьшением медиан общей (OB) и безрецидивной выживаемости (БРВ): 11,3 vs 15,8 мес. при FLT3-ITD( $p=0,005)$ и 10 vs 13,3 мес. при FLT3-ITD+ ( $p=0,009)$ соответственно. Также оценивали связь уровня аллельной нагрузки FLT3-ITD с длительностью OB. В группе с

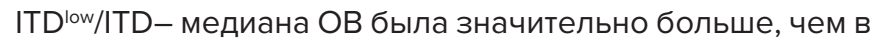
группе c ITD high ( $p=0,028)$. Группа пациентов с 1 мутацией в гене NPM1 имела значительно лучшие ОВ и БРВ по

\section{MYELOID TUMORS}

EV Motyko', OV Blau², LB Polushkina', LS Martynenko', MP Bakai', NYu Tsybakova', YuS Ruzhenkova', EV Kleina', NB Pavlenko', AM Radzhabova1, EV Karyagina ${ }^{3}$, OS Uspenskaya 4 , SV Voloshin', AV Chechetkin', IS Martynkevich ${ }^{1}$

${ }^{1}$ Russian Research Institute of Hematology and Transfusiology, 16 2-ya Sovetskaya str., Saint Petersburg, Russian Federation, 191024

${ }^{2}$ Charite Clinic, Berlin Medical University,

30 Hindenburgdamm, Berlin, Germany, 12200

${ }^{3}$ Municipal Hospital No. 15, 4 Avangardnaya str., Saint Petersburg, Russian Federation, 198205

${ }^{4}$ Leningrad Regional Clinical Hospital, 45-49 Lunacharskogo pr-t, Saint Petersburg, Russian Federation, 194291

\section{ABSTRACT}

Aim. To analyze the effect on prognosis of mutations that are typical of acute myeloid leukemia (AML) patients.

Materials \& Methods. The study included 620 AML patients surveyed at Hematology Clinics of Saint Petersburg (Russia) and Charite Clinic (Berlin, Germany). G-banding of chromosomes was employed for cytogenetic testing. Aberration screening in DNMT3A, IDH1/2 genes was based on real-time polymerase chain reaction (PCR) with subsequent analysis of melting and sequencing profiles. Mutations in FLT3, NPM1 genes were revealed by PCR.

Results. Mutations were identified in 343 (55.3\%) out of 620 patients. Significantly more often mutations were discovered in patients with normal karyotype (NK) $(p=0.001)$. FLT3-ITD mutation was associated with reduced medians of overall survival (OS) and disease-free (DFS) survival: 11.3 vs. 15.8 months with FLT3-ITD- $(p=0.005)$ and 10.0 vs. 13.3 months with FLT3-ITD+ $(p=0.009)$, respectively. The relation of FLT3-ITD allele burden to OS duration was also assessed. In the ITD ${ }^{\text {low }} /$ ITD - group the OS median was considerably longer than in the ITD ${ }^{\text {high }}$ group $(p=0.028)$. In the group of patients with 1 mutation in NPM1 gene OS and DFS were much better in comparison with other patients (medians of 27.4 and 13.9 months, respectively, $p=0.040 ; 19.3$ and 12.0 months, $p=0.049$ ). Negative impact of mutations in DNMT3A gene was noticed while assessing OS median: 12 (DN$M T 3 A+)$ and 15 months (DNMT3A-), respectively $(p=0.112)$. 
сравнению с другими больными (медианы соответственно 27,4 и 13,9 мес., $p=0,040 ; 19,3$ и 12 мес., $p=0,049$ ). Тенденция к негативному влиянию мутаций в гене DNMT3A прослеживалась при оценке медианы ОВ: 12 (DNMT3A+) и 15 мес. (DNMT3A-) соответственно ( $p=0,112)$. Наличие мутаций в гене IDH1 коррелировало с лучшей OB по сравнению с группой без мутаций ( $p=0,092)$. Полиморфизм rs11554137 в гене IDH1 был связан с ухудшением ОВ в группе больных с НK ( $p=0,186)$. У 144 пациентов обнаружены различные комбинации мутаций - от 2 до 5. Показано, что мутации в генах FLT3 (FLT3-ITD), NPM1, DNMT3A и IDH2 статистически значимо чаще выявлялись в сочетании с другими мутациями ( $p=0,001)$ : NPM1+/ FLT3-ITD+ (20,8 \%), NPM1+/FLT3-ITD+/DNMT3A+ (8,3 \%) и FLT3-ITD+/DNMT3A+ (8,3 \%). Больные с 1 мутацией имели значительно большую медиану ОВ по сравнению с пациентами с 2 мутациями (18,1 и 12,2 мес.; $p=0,003)$. у больных с NPM1+ наиболее неблагоприятной по показателям ОВ дополнительной мутацией была FLT3-ITD (медиана 27,4 vs 9,2 мес.; $p=0,019$ ), а также сочетание NPM1+/FLT3-ITD+/DNMT3A+ (медиана 27,4 vs 14,6 мес.; $p=0,141)$. Пациенты с DNMT3A+ имели тенденцию к ухудшению ОВ при наличии дополнительной мутации FLT3ITD (17,3 vs 7,1 мес.; $p=0,074)$.

Заключение. Мутации в генах FLT3, DNMT3A, IDH1/2, NPM1 часто встречаются у больных ОМЛ промежуточного риска, т. е. они определяют группу промежуточного прогноза при ОМЛ. Исследованные мутации существенно влияют на прогноз, при этом важно учитывать тип мутации, ее аллельную нагрузку и наличие дополнительных мутаций. Наличие 2 мутаций у 1 больного значительно снижает ОВ по сравнению с пациентами с 1 мутацией. Худший прогноз имеют больные из исследованной группы с сочетанием мутаций NPM1+/FLT3-ITD+, NPM1+/FLT3-ITD+/DNMT3A+, DNMT3A+/FLT3-ITD+. Комплексный анализ генетических повреждений у больных ОМЛ позволяет наиболее точно определять прогноз течения заболевания и планировать проведение целенаправленной терапии.

Ключевые слова: острые миелоидные лейкозы, мутации генов FLT3, NPM1, DNMT3A, IDH1/2, кариотип, прогноз.

Получено: 13 июля 2018 г.

Принято в печать: 16 января 2019 г.

Для переписки: Екатерина Вадимовна Мотыко, канд. биол. наук, ул. 2-я Советская, д. 16, Санкт-Петербург, Российская Федерация, 191024; тел.: +7(812)925-05-62; e-mail: genetics.spb@mail.ru

Для цитирования: Мотыко Е.В., Блау О.В., Полушкина Л.Б. и др. Прогностическое значение генетических мутаций у больных острыми миелоидными лейкозами: результаты совместного исследования гематологических клиник Санкт-Петербурга (Россия) и клиники Шарите (Германия). Клиническая онкогематология. 2019;12(2):211-9.

DOI: 10.21320/2500-2139-2019-12-2-211-219
Mutations in IDH1 gene correlated with a better OS than in the group without mutations $(p=0.092)$. The rs 11554137 polymorphism in $I D H 1$ gene was associated with worse OS in the group of patients with NK $(p=0.186)$. In 144 patients various mutation combinations (from 2 to 5 ) were identified. It was demonstrated that mutations in FLT3 (FLT3-ITD), NPM1, $D N M T 3 A$, and IDH2 were identified significantly more often in combinations with other mutations $(p=0.001)$ : NPM1+/ FLT3-ITD+ (20.8\%), NPM1+/FLT3-ITD+/DNMT3A+ (8.3\%), and FLT3-ITD+/DNMT3A+ (8.3\%). Patients with 1 mutation had a noticeably longer OS median compared with patients with 2 mutations (18.1 and 12.2 months; $p=0.003$ ). In patients with NPM1+ according to their OS the most unfavorable additional mutation was FLT3-ITD (median 27.4 vs. 9.2 months; $p=0.019)$ and the combination of NPM1+/FLT3-ITD+/DNMT3A+ (median 27.4 vs. 14.6 months; $p=0.141$ ). OS of patients with DNMT3A+ showed a downward trend if FLT3-ITD additional mutation was identified (17.3 vs. 7.1 months; $p=0.074$ ). Conclusion. Mutations in FLT3, DNMT3A, IDH1/2, NPM1 genes frequently occur in $A M L$ intermediate-risk patients, i.e. they determine the intermediate prognosis group in AML. The studied mutations considerably impact prognosis. It is important to take into consideration mutation type, its allele burden, and the presence of additional mutations. A patient with 2 mutations has a considerably worse OS compared with a patient with 1 mutation. The studied group of patients with the combination of NPM1+/FLT3-ITD+, NPM1+/ FLT3-ITD+/DNMT3A+, DNMT3A+/FLT3-ITD+ mutations has the poorest prognosis. Comprehensive analysis of genetic damages in AML patients allows to most accurately predict the course and prognosis of the disease and to plan targeted therapy.

Keywords: acute myeloid leukemias, mutations in FLT3, NPM1, DNMT3A, IDH1/2 genes, karyotype, prognosis.

Received: July 13, 2018

Accepted: January 16, 2019

For correspondence: Ekaterina Vadimovna Motyko, PhD in Biology, 16 2-ya Sovetskaya str., Saint Petersburg, Russian Federation, 191024; Tel.: +7(812)925-05-62; e-mail: genetics.spb@mail.ru

For citation: Motyko EV, Blau OV, Polushkina LB, et al. Prognostic Value of Genetic Mutations in Patients with Acute Myeloid Leukemias: Results of a Cooperative Study of Hematology Clinics of Saint Petersburg (Russia) and Charite Clinic (Germany). Clinical oncohematology. 2019;12(2):211-9 (In Russ).

DOI: 10.21320/2500-2139-2019-12-2-211-219

\section{ВВЕДЕНИЕ}

Острые миелоидные лейкозы (ОМЛ) - это злокачественные опухоли системы крови, характеризующиеся экстенсивной пролиферацией незрелых миелоидных клеток в сочетании с нарушенной дифференцировкой $[1,2]$. В то время как при различных гематологических заболеваниях в течение длительного времени успешно применяется таргетная терапия (ингибиторы 
тирозинкиназ при хроническом миелоидном лейкозе, ибрутиниб при хроническом лимфоцитарном лейкозе и т. д.), индукционная терапия при ОМЛ существенно не менялась за последние несколько десятилетий [3, 4]. Исключением стал острый промиелоцитарный лейкоз, при котором использование полностью транс-ретиноевой кислоты позволило преодолеть блок в миелоидной дифференцировке, вызываемый химерным геном $P M L$ $R A R \alpha$. Современная терапия ОМЛ позволяет достичь полной ремиссии примерно у $75 \%$ молодых (<60 лет) пациентов. Однако в дальнейшем у большинства из них развиваются рецидивы [5]. Такое клиническое течение ОМЛ объясняется наличием популяции лейкозных клеток, которая обусловливает развитие рецидивов при ОМЛ даже после достижения полной ремиссии.

Изначально в соответствии с Франко-американобританской классификацией (FAB) больных ОМЛ распределяли на 8 подгрупп (М0-M7), основываясь в основном на морфоцитохимических особенностях опухолевых клеток [6]. Внедрение цитогенетических исследований позволило выделить три группы риска: благоприятный, промежуточный и неблагоприятный прогноз. Так, по результатам кариотипирования клиницисты относили, например, пациентов с CBFлейкозами [cinv(16)/t(16;16) или $t(8 ; 21)]$ в группу благоприятного прогноза и назначали терапию только высокими дозами цитарабина, достигая удовлетворительных и долгосрочных лечебных результатов. $\mathrm{y}$ пациентов с высоким риском (моносомный или комплексный кариотип) проведения одной химиотерапии недостаточно и следует сразу начинать поиск донора на трансплантацию аллогенных гемопоэтических стволовых клеток (аллоТГСК). Однако 40-50\% пациентов с ОМЛ имеют нормальный кариотип (НК), т. е. относятся к промежуточной группе риска, а показатели их выживаемости и ответ на терапию сильно варьируют [7]. Это послужило основанием для расширения классификации и включения в качестве дополнительных факторов прогноза мутаций генов, характерных для пациентов с ОМЛ.

К настоящему времени использование метода полногеномного секвенирования позволило получить наиболее широкое представление о возникновении и развитии лейкозного клона. Однако в целом эти знания только начинают использоваться при разработке новых лечебных стратегий и распределении пациентов в группы риска. Внедрение в алгоритм диагностики больных ОМЛ нескольких молекулярных маркеров, таких как мутации в генах FLT3, NPM1 и $C E B P A$, значительно повлияло на прогностическую стратификацию НК-ОМЛ. Стало очевидно, что индивидуализированное и наиболее глубокое изучение биологических характеристик и свойств лейкозных клеток позволит оптимизировать стратегию лечения пациентов с ОМЛ, особенно на этапе консолидации. Тщательное исследование наличия молекулярногенетических маркеров у больных ОМЛ послужит дополнительным аргументом в выборе кандидатов на аллоТГСК, учитывая сопутствующие риски или группы пациентов, у которых будет достаточно применения только химиотерапии.

Ранее предполагалось, что в основе патогенеза ОМЛ лежит двухступенчатый процесс, который включает появление на начальном этапе мутаций I класса, инициирующих митоген-независимую пролиферацию и выживаемость лейкозных клеток, а затем - мутаций II класса, которые воздействуют на регуляцию транскрипции, блокируя дифференцировку клеток [8]. Однако сейчас можно утверждать, что в патогенезе ОМЛ задействован более широкий спектр повреждений генов с разной функциональной нагрузкой [9-11]. Рецепторы тирозинкиназ, например FLT3, регулируют пролиферацию, дифференцировку и выживание нормальных гемопоэтических стволовых клеток, а мутации приводят к конститутивной активации рецепторов. Мутация FLT3-ITD (внутренняя тандемная дупликация) присутствует почти в $1 / 3$ случаев ОМЛ и связана с неблагоприятным прогнозом, включая повышенный риск развития рецидивов и ухудшение общей выживаемости (OB) [12]. Наиболее значительное негативное влияние мутации FLT3-ITD показано у больных с НК, особенно у пациентов с высокой аллельной нагрузкой мутации [13]. Помимо того, что FLT3-ITD является важным прогностическим маркером, в настоящее время данная мутация стала мишенью для таргетной терапии. Показано, что ингибитор тирозинкиназы мидостаурин способен улучшать ОВ и бессобытийную выживаемость при назначении одновременно со стандартной индукционной и консолидирующей терапией ОМЛ [14]. Нуклеофосмин B23 NPM1 расположен на хромосоме 5 (5q35) и кодирует многофункциональный фосфопротеид. Мутации приводят к его переходу из ядра в цитоплазму. Многие исследователи описывают благоприятный прогноз у пациентов с мутациями в гене NPM1, однако следует учитывать сильное влияние дополнительных к NPM1+ мутаций, например FLT3-ITD и/или в гене DNMT3A, которые значительно ухудшают выживаемость больных оМЛ [15-17].

Возвращаясь к механизмам патогенеза ОМЛ, можно заключить, что изменения обнаруживаются как на генетическом, так и эпигенетическом уровне. В настоящее время значение генетических аберраций при ОМЛ достаточно хорошо изучено, а эпигенетические нарушения стали предметом пристального внимания относительно недавно. Основанием для этого стало в т. ч. внедрение в клиническую практику гипометилирующих препаратов: децитабина и азацитидина [18]. DNMT3A и IDH1/2 участвуют в регуляции метилирования нуклеотидных остатков в составе ДНК и процессов клеточного метаболизма. Мутации генов приводят к нарушению данных процессов и изменению экспрессии генов. Прогностическое значение этих мутаций противоречиво. Некоторые исследователи не обнаружили значительного влияния на прогноз мутаций в генах $D N M T 3 A$ и $I D H 1 / 2$, тогда как есть данные о корреляции мутаций в генах DNMT3A и IDH1 с неблагоприятным прогнозом. При этом важную роль играли такие биологические факторы, как кариотип, возраст и наличие дополнительных мутаций [19-24].

Таким образом, очевидно, что ОМЛ является генетически вариабельным, сложным, динамическим заболеванием. Приоритетная задача на сегодня создание новой системы распределения больных по группам риска, основанной как на результатах цитогенетического исследования, так и на влиянии 
дополнительных молекулярных повреждений и их комбинаций на прогноз. В настоящей статье мы анализируем значение наиболее частых мутаций при ОМЛ и их сочетаний для прогнозирования течения заболевания, выявления риска развития рецидивов и подбора адекватной терапии.

\section{МАТЕРИАЛЫ И МЕТОДЫ}

В исследование включено 620 пациентов с ОМЛ: 451 из них проходили обследование в клинике Шарите (Берлин, Германия), а 169 - в гематологических клиниках Санкт-Петербурга. Больные были в возрасте 16-85 лет (медиана 57 лет); 330 (53,2 \%) мужчин и 290 (46,8 \%) женщин. У 483 (77,9 \%) больных верифицирован de nоvo ОМЛ, у 137 (22,1 \%) - вторичный ОМЛ из предшествующих миелодиспластического синдрома или лимфомы.

Цитогенетический анализ выполняли на G-дифференциально окрашенных хромосомах, интерпретацию проводили в соответствии с Международной номенклатурой дифференциально сегментированных хромосом (ISCN, 2013). В каждом исследовании было проанализировано не менее 20 метафазных пластин.

Для исследования мутационного статуса генов FLT3, NPM1, DNMT3A, IDH1/2 использовали геномную ДНК и РНК. Выделение геномной ДНК из периферической крови (ПК) или костного мозга (КМ) проводили с помощью метода хлороформной экстракции. Выделение РНК выполняли с использованием наборов «АмплиСенс Лейкоз Квант» («Интерлабсервис», Россия). Праймеры для полимеразной цепной реакции (ПЦР) подбирали с помощью программы VectorNTI. Haличие внутренней тандемной дупликации в гене FLT3 (FLT3-ITD) идентифицировали на электрофореграмме (6\% полиакриламидный гель). Определение точечной мутации FLT3-TKD (замена аспарагина в положении 835) проводили методом ПЦР с последующим анализом длин рестрикционных фрагментов (эндонуклеаза рестрикции EcoRV, Thermo Fisher Scientific). Анализ наличия инсерций (в основном, четырех пар нуклеотидов) в гене NPM1 выполнен путем ПЦР с обратной транскрипцией и визуализации продуктов реакции на электрофорезе. Скрининг аберраций в генах DNMT3A, IDH1/2 осуществляли методом ПЦР в режиме реального времени с дальнейшим анализом кривых плавления и определением варианта мутации методом секвенирования амплифицированного фрагмента. Фрагментный анализ FLT3-ITD проводили с помощью ПЦР путем использования прямого праймера с флюоресцентной меткой и анализатора Applied Biosystems.
Статистический анализ выполнен с использованием компьютерной программы Statistica 10.0. Непараметрические данные сравнивали путем построения таблиц сопряженности признаков по критерию Пирсона $\chi^{2}$. Для анализа выживаемости строили кривые по методу Каплана-Мейера. Для сравнения выживаемости в различных группах использовали двусторонний лог-ранговый тест. Для анализа безрецидивной выживаемости (БРВ) длительность ремиссии рассчитывали от начала лечения до возникновения рецидива заболевания, а ОВ - от начала лечения до даты построения кривых или смерти больного. Во всех случаях различия между группами считались статистически значимыми при $p<0,05$.

\section{РЕЗУЛЬТАТЫ}

В зависимости от обнаруженных цитогенетических поломок пациенты распределены в следующие прогностические группы: 417 (67,2 \%) больных — группа промежуточного прогноза, 68 (11\%) - благоприятного, 135 (21,8 \%) - неблагоприятного. НК был у 328 $(52,9 \%)$ из 620 пациентов. Мутации обнаружены у 343 (55,3 \%) из 620 участников. Одиночные повреждения генов определялись у 199 (58 \%) больных, остальная группа имела различные комбинации мутаций: 2 мутации - у 99 больных, 3 мутации - у 31, 4 мутации у 13 и 5 мутаций - у 1. Наиболее часто наблюдали следующие сочетания мутаций: NPM1+/FLT3-ITD+ (30/144, 20,8 \%), NPM1+/FLT3-ITD+/DNMT3A+ (12/144, $8,3 \%)$ и FLT3-ITD+/DNMT3A+ (12/144, 8,3 \%) (рис. 1). Показано, что мутации в генах FLT3 (FLT3-ITD), NPM1, $D N M T 3 A$ и IDH2 статистически значимо чаще выявлялись в сочетании с другими мутациями $(p=0,001)$, а не одиночно. Статистически значимо чаще мутации определялись у больных с НК - у 220 из 328 изученных нами пациентов с НК $(p=0,001)$. В группе с неблагоприятным кариотипом мутации выявлены только у 50 из 135 больных (рис. 2).

Мутации в генах FLT3 и NPM1 наиболее часто встречались в исследуемой группе больных ОМЛ (внутренние тандемные дупликации FLT3-ITD - у 23,2 \% пациентов, точечная замена FLT3-TKD - у 6,6 \%, инсерции в экзоне 12 гена NPM1 - у 21,8 \%). Мутации в экзоне 23 (чаще в кодоне 882) гена DNMT3A выявлены у 14,2 \% больных, в экзоне 4 гена IDH1 - у $15,6 \%$ (в основном, IDH1R132 и однонуклеотидный полиморфизм rs11554137), в экзоне 4 гена IDH2 - у 6,8 \% (в основном, в локусах R140 и R172).

Есть данные о важной роли соотношения мутантного аллеля ITD и аллеля дикого типа (ITD-AR), поэтому мы разделили больных с мутациями FLT3-ITD на

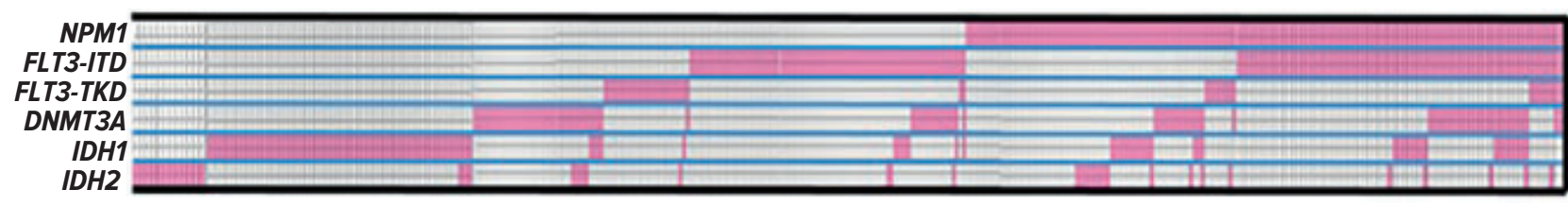

Рис. 1. Частота мутаций в генах FLT3, NPM1, DNMT3A, IDH1/2 у больных ОМЛ 
две группы (пороговый уровень 0,5): с низким $(<0,5)$ и высоким $(\geq 0,5)$ уровнем аллельной нагрузки. Таким образом, 83 (57,6 \%) пациента имели низкий уровень аллельной нагрузки (ITD $\left.{ }^{\mathrm{low}}\right)$ и $61(42,4 \%)$ - высокий $\left(\right.$ ITD $\left.^{\text {high }}\right)$. У больных с ITD $^{\text {high }}$ обнаружено увеличение количества лейкоцитов в ПК и доли бластных клеток в КМ по сравнению с больными с ITD ${ }^{\text {low: }} 71,1 \times 10^{9} /$ л и $39,3 \times 10^{9} / л(p=0,002), 90,2$ и $81,1 \%(p=0,052)$ соответственно. У пациентов с ITD ${ }^{\text {high }}$ статистически значимо чаще обнаруживался полиморфизм rs11554137 в гене IDH1 по сравнению с больными с $\operatorname{ITD}^{\text {low }}(p=0,008)$. Медианы ОВ больных с ITD $^{\text {high }}$, ITD $^{\text {low }}$ и ITD- распределились соответственно как 10,4, 11,9 и 15,8 мес. ( $p=0,016)$; для БРВ - 9,1, 10,4 и 13,3 мес. соответственно $(p=0,032)$. При этом статистически значимых отличий ОВ и БРВ между группами ITD $^{\text {high }}$ и ITD $^{\text {low }}$ не наблюдалось. Только при объединении ITD ${ }^{\text {low }}$ и ITD- в общую группу удалось выявить значимое ее отличие в длительности медианы ОВ от больных с $\operatorname{ITD}^{\text {high }}(p=0,028)$ (рис. 3). При сравнении прогноза у пациентов с FLT3-TKD ${ }^{\text {high }}$ и FLT3-TKD ${ }^{\text {low }}$ обнаружена тенденция к ухудшению ОВ у больных с высокой аллельной нагрузкой мутации FLT3-TKD (медиана 7,4 и 9 мес. соответственно; $p=0,220$ ).

Больные с мутацией FLT3-ITD были моложе больных без мутации (медиана возраста составила $56,8$ и 60,1 года соответственно; $p=0,021)$. Мутации FLT3-TKD чаще обнаруживались у больных с M5-вариантом ОМЛ ( $p=0,032)$, а мутации FLT3-ITD у пациентов с М2- и М3-вариантами ОМЛ ( $p=0,248$ и $p=0,289$ соответственно). Мутации FLT3-ITD редко встречались у больных со вторичными ОМЛ $(p=0,002)$. У больных с мутациями FLT3-ITD и FLT3-TKD обнаруживался высокий уровень лейкоцитов по сравнению с пациентами без мутации: $56,2 \times$ $10^{9} /$ л и $7 \times 10^{9} /$ л $(p=0,001), 33,1 \times 10^{9} /$ л и $10,2 \times 10^{9} /$ л ( $p=0,002)$ соответственно. У пациентов с FLT3-ITD доля бластных клеток в КМ была выше по сравнению с пациентами без мутации - 90,3 и 80,1 \% соответственно $(p=0,001)$.

При сопоставлении данных цитогенетического и молекулярно-генетического исследований получены следующие результаты. У 71,5 \% пациентов с мутацией FLT3-ITD определялся НК ( $p=0,001)$ и только у 6,9 \% - неблагоприятный и благоприятный кариотипы ( $p=0,001$ и $p=0,078$ соответственно). $\mathrm{y}$ пациентов с FLT3-TKD чаще выявляли промежуточный кариотип ( $p=0,238)$. Мутации в гене FLT3 преимущественно встречались в сочетании с мутациями в других исследованных генах. Так, мутации FLT3-ITD и FLT3-TKD часто обнаруживались в сочетании с мутациями в гене NPM1+ ( $p=0,001$ и $p=0,017$ соответственно), а также FLT3-ITD+ с DNMT3A+ $(p=0,001)$. Мутации FLT3-TKD не найдены в комбинации с мутациями в гене IDH1 ни у одного больного $(p=0,05)$. Медианы OB и БРВ пациентов с FLT3-ITD по сравнению с больными без мутации составили 11,3 и 15,8 мес., 10 и 13,3 мес. соответственно ( $p=0,005$ и $p=0,009$ соответственно) (рис. 4).

Мутации в гене NPM1 были связаны с M5-вариантом ОМЛ $(p=0,024)$ и редко выявлялись у больных со вторичными лейкозами ( $p=0,001)$. Статистически значимо чаще мутации находили у пациентов с проме-

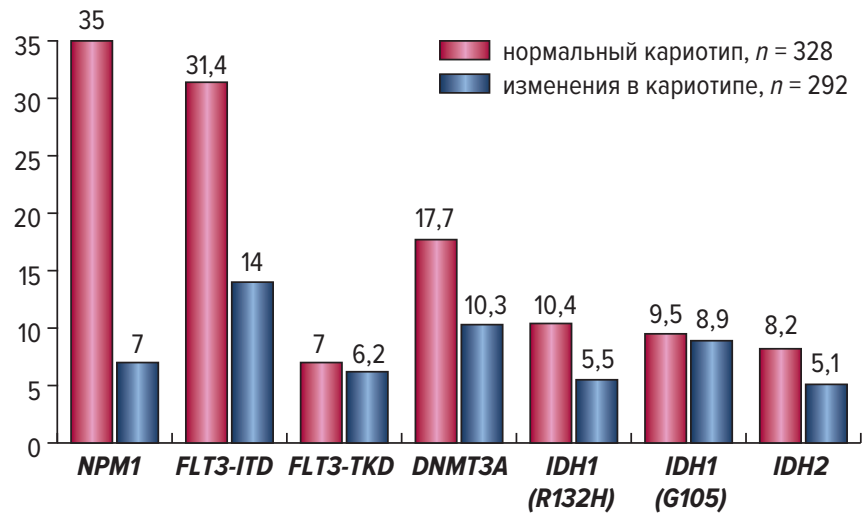

Рис. 2. Частота мутаций (в \%) в генах FLT3, NPM1, DNMT3A, IDH1/2 у пациентов с нормальным кариотипом и хромосомными аномалиями

Fig. 2. Mutation rate in FLT3, NPM1, DNMT3A, IDH1/2 genes in patients with normal karyotype and chromosomal abnormalities (in \%)

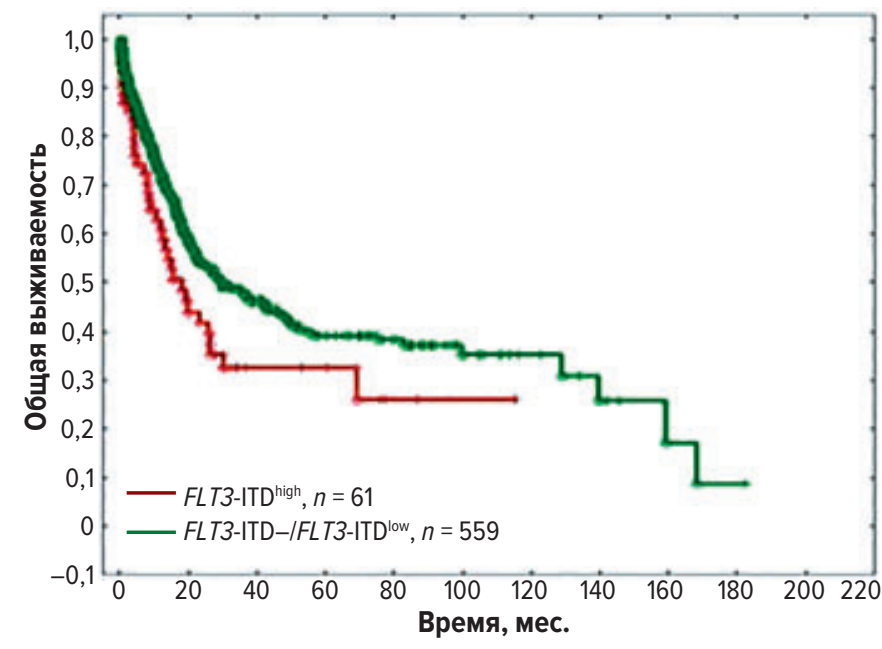

Рис. 3. Общая выживаемость больных ОМЛ в группах с различным уровнем аллельной нагрузки FLT3-ITD ( $p=0,028)$

Fig. 3. Overall survival of AML patients in groups with different FLT3ITD allele levels $(p=0.028)$

жуточным прогнозом в соответствии с результатами кариологического анализа $(133 / 135 ; 98,5 \% ; p=0,001)$. У пациентов с NPM1+ преимущественно выявлялись дополнительные мутации: FLT3-ITD, DNMT3A+, IDH1+, $I D H 2+$ (особенно в локусе R140) ( $p=0,001$ для всех указанных), FLT3-TKD ( $p=0,017)$. У пациентов с NPM1+ статистически значимо чаще достигались полные ремиссии (ПР) по сравнению с больными без мутации в гене NPM1 - 94,8 и 83,1 \% соответственно $(p=0,001)$. Медианы ОВ и БРВ у больных с NPM1+ были выше, чем у пациентов без мутации, - 16,8 и 13,6 мес., 13,3 и 12,2 мес. соответственно, однако различия не были статистически значимыми ( $p=0,485$ и $p=0,352$ соответственно). Наибольшее благоприятное влияние наличие мутаций в гене NPM1 установлено в группе больных в возрасте 60 лет и менее: медиана ОВ составила 22,7 (NPM1+) и 19,1 мес. (NPM1-) ( $p=0,184)$. Кроме того, в группе пациентов с 1 мутацией NPM1+ отмечались лучшие показатели ОВ и БРВ по сравнению с другими больными (27,4 и 13,9 мес., $p=0,040$ и 19,3 и 12 мес., $p=0,049$ соответственно) (рис. 5). 

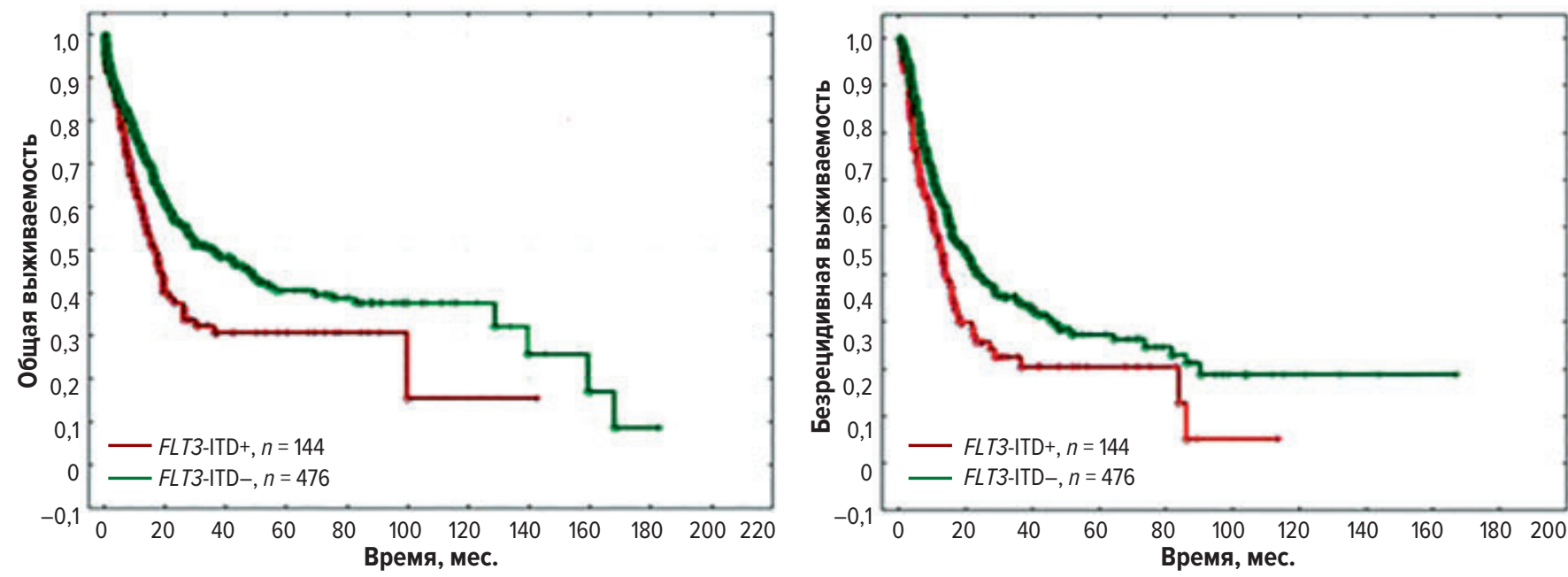

Рис. 4. Прогностическое значение мутаций FLT3-ITD у больных ОМЛ: общая $(p=0,005)$ и безрецидивная выживаемость $(p=0,009)$

Fig. 4. Prognostic value of FLT3-ITD mutations in AML patients: overall survival $(p=0.005)$ and disease-free survival $(p=0.009)$
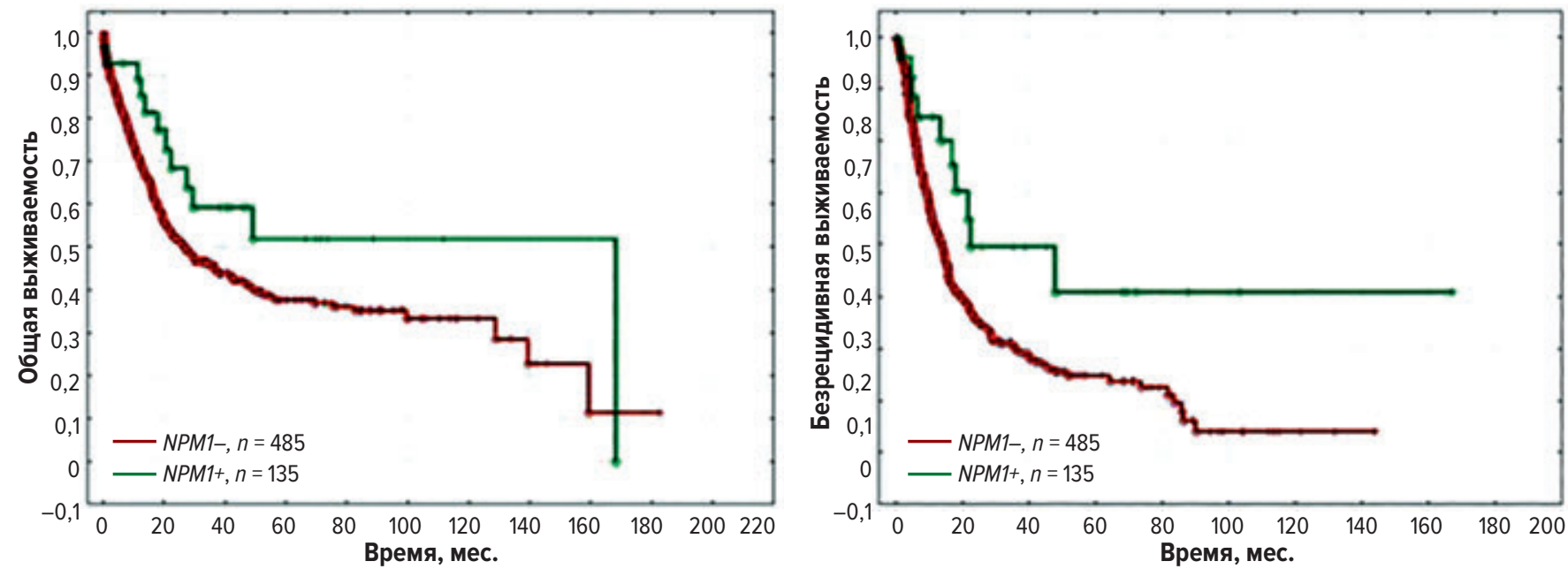

Рис. 5. Прогностическое значение одиночной мутации в гене NPM1 у больных ОМЛ: общая $(p=0,040)$ и безрецидивная выживаемость $(p=0,049)$

Fig. 5. Prognostic value of a single mutation in NPM1 gene in AML patients: overall survival $(p=0.040)$ and disease-free survival $(p=0.049)$

Показана тенденция к увеличению возраста больных с мутацией в гене DNMT3A $(62,1$ vs 59 лет у пациентов без мутаций; $p=0,138)$. В дебюте заболевания пациенты с DNMT3A+ имели высокий уровень лейкоцитов в ПК и бластных клеток в КМ по сравнению с больными без мутации $\left(38,1 \times 10^{9} /\right.$ л и $9 \times 10^{9} /$ л, $p=0,001 ; 81,4$ и $80,0 \%, p=0,111$ соответственно). Мутации в гене DNMT3A были связаны с М4- и М5-вариантами ОМЛ ( $p=0,001$ и $p=0,015$ соответственно). Статистически значимо чаще мутации встречались у больных с НК $(p=0,001)$ и в сочетании с мутациями FLT3-ITD, NPM1+ $(p=0,001)$ и IDH2+R140 $(p=0,056)$. Негативное влияние (тенденция) мутаций в гене DNMT3A четко прослеживалось при оценке медианы ОВ пациентов, которая составила 12 (DNMT3A+) и 15 мес. (DNMT3A-) соответственно $(p=0,112)$.

Больных с аберрациями в гене IDH1 разделили на две группы: с мутациями (в основном, в локусе R132) и с однонуклеотидным полиморфизмом rs11554137 (G105). Мутации в гене IDH2 встречались в основном в локусах R140 ( $n=36)$ и R172 $(n=3)$, y
3 пациентов - в других кодонах. Пациенты с мутациями в генах IDH1 и IDH2 были старше больных без мутаций $(62,1$ vs 60 лет, $p=0,147$ и 63 vs 59,2 года соответственно; $p=0,069)$. Количество лейкоцитов в ПК было статистически значимо меньше у больных с $I D H 1+\left(5,1 \times 10^{9} /\right.$ л и $12 \times 10^{9} /$ л; $\left.p=0,003\right)$. У большинства пациентов с IDH1+ и IDH2+ был НК ( $p=0,026$ и $p=0,126$ соответственно). Имела место тенденция к увеличению количества пациентов, достигнувших ПР, в группе с IDH1+ по сравнению с группой без мутации (92,0 и 83,1\% соответственно; $p=0,181)$. Наличие мутаций в гене IDH1 коррелировало с лучшей OB по сравнению с группой без мутаций (медиана 19,3 и 14,3 мес.; $p=0,092$ ), тогда как наличие полиморфизма rs11554137 в гене IDH1 было связано с тенденцией к ухудшению ОВ в группе больных с НК (медиана 12,2 и 15,5 мес.; $p=0,186$ ).

В связи с тем, что примерно у половины больных выявлено наличие нескольких мутаций в разных генах, причем прогностическое значение этих мутаций могло быть прямо противоположным, мы изучили, 
как влияют на прогноз сочетания некоторых мутаций. Для анализа выбрали мутации NPM1+, FLT3-ITD и DNMT3A+, которые наиболее отчетливо влияли на прогноз и часто встречались в сочетании с другими аберрациями. На рис. 6 показаны графики ОВ при различных сочетаниях мутаций. Выявлено, что больные, у которых находили 1 мутацию, имели значительно лучшую ОВ по сравнению с пациентами с 2 мутациями (медиана 18,1 и 12,2 мес.; $p=0,003$ ). Таким образом, наличие любой дополнительной мутации к NPM1+, FLT3-ITD и DNMT3A+ ухудшало медиану OB. При этом у больных с NPM1+ наиболее неблагоприятной дополнительной мутацией была FLT3-ITD (медиана OB 27,4 мес. для NPM1+/другие изучавшиеся в работе мутации отсутствуют и 9,2 мес. для NPM1+/FLT3-ITD+; $p=0,019)$, а также сочетание NPM1+/FLT3-ITD+/ $D N M T 3 A+$ (медиана OB 14,6 мес.; $p=0,141)$. Пациенты c DNMT3A+ имели худшую ОВ при наличии мутации FLT3-ITD (медиана OB 17,3 мес. для DNMT3A+/другие изучавшиеся в работе мутации отсутствуют и 7,1 мес. для DNMT3A+/FLT3-ITD+; $p=0,074)$. У больных с FLT3-ITD наличие дополнительных мутаций значимой роли не играло.

\section{ОБСУЖДЕНИЕ}

В настоящее время информация о прогностическом значении молекулярно-генетических аберраций активно используется в клинической практике. В 2017 г. Европейская сеть по изучению лейкозов (ELN) представила новую классификацию ОМЛ с расширенной панелью генетических мутаций, которую можно применять для предсказания течения болезни. Однако, учитывая геномную гетерогенность заболевания, необходимо продолжать исследования различных групп пациентов с целью изучить клиническое значение всей сложной генетической архитектуры заболевания. Мы проанализировали данные 620 больных ОМЛ. В соответствии с предыдущими исследованиями [25] мутации в анализируемых генах у больных ОМЛ встречаются часто - более 10 \% в генах NPM1, FLT3, DNMT3A. Пациенты с благоприятным и неблагоприятным кариотипами и, в частности, со сбалансированными перестройками значительно реже имели мутации по сравнению с лицами из группы промежуточного риска и с НК, что согласуется с данными литературы [26].

По нашим данным, наличие мутации FLT3-ITD связано с высоким уровнем лейкоцитов в ПК и бластных клеток в КМ, негативным влиянием на ОВ и риском развития рецидивов. Однако, несмотря на то что многочисленные исследования $[12,27,28]$ подтверждают эту информацию, сейчас большое внимание уделяется таким особенностям, как уровень аллельной нагрузки мутации FLT3-ITD и размер инсерции [29-31]. Мы также обнаружили связь уровня аллельной нагрузки FLT3-ITD с длительностью медианы OB: продолжительность жизни в группе больных с FLT3-ITD ${ }^{\text {low }} /$ FLT3-ITD- была значительно больше, чем с FLT3-ITD ${ }^{\text {high }}$. Пациентам с FLT3-ITD $^{\text {high }}$ рекомендована аллоТГCК в ПР, которая значительно улучшает БРВ и ОВ [32, 33].

У 42 \% пациентов были выявлены различные комбинации мутаций, причем наиболее часто встречались
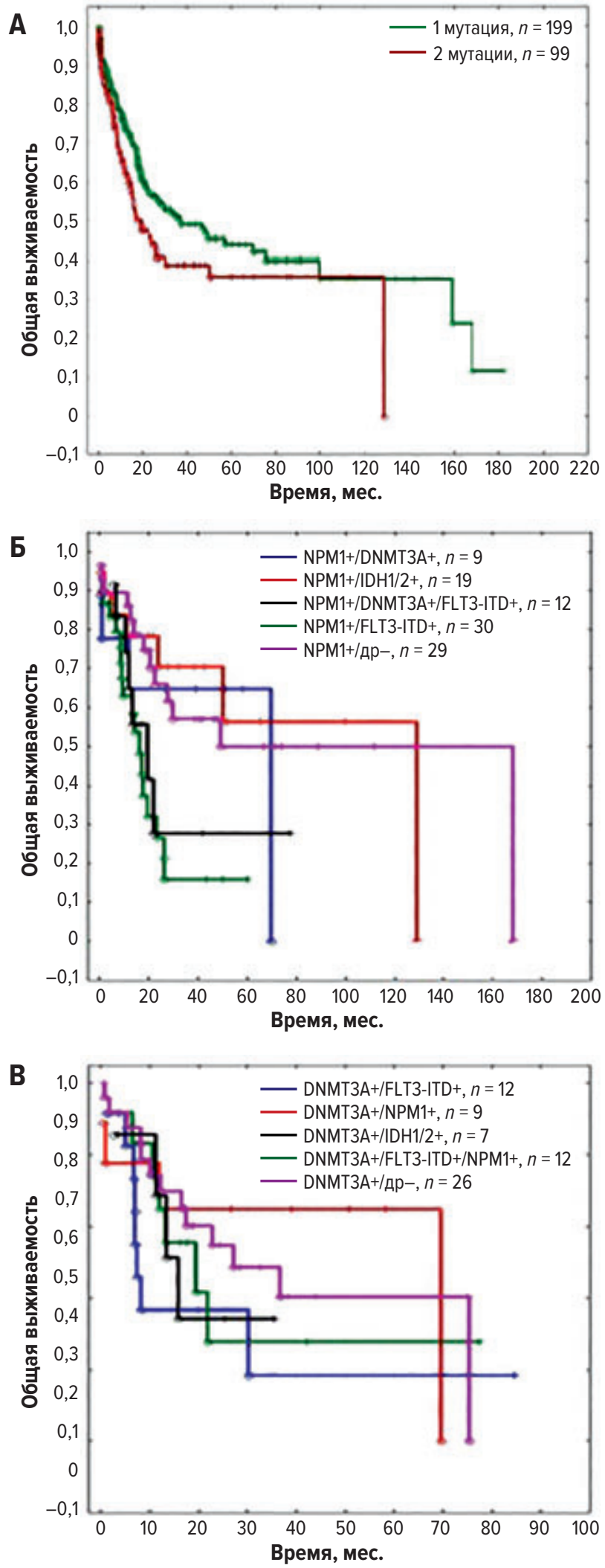

Рис. 6. Общая выживаемость больных ОМЛ и влияние дополнительных мутаций на прогноз:

$A-$ c 1 и 2 мутациями ( $p=0,003) ; 5-$ с мутациями в гене NPM1 (FLT3-ITD, $p=0,019 ;$ FLT3-ITD+/DNMT3A+, $p=0,141) ; B-$ с мутациями в гене DNMT3A ( $p=0,074)$

др - другие изучавшиеся мутации.

Fig. 6. Overall survival of AML patients and effect of additional mutations on prognosis:

$A-$ with 1 and 2 mutations $(p=0.003) ; E-$ with mutations in NPM1 gene (FLT3-ITD, $p=0.019$; FLT3-ITD+/DNMT3A+, $p=0.141$ ); $B-$ with mutations in DNMT3A gene $(p=0.074)$

др - other mutations under analysis. 
сочетания мутаций в гене DNMT3A с мутациями в генах NPM1 и FLT3, а также FLT3-ITD+/NPM1+, что коррелирует с данными литературы [34]. Наличие нескольких мутаций у 1 больного, их сочетания или их взаимное исключение являются важным объектом исследований в настоящее время, т. к. данная информация позволяет наиболее полно представить патогенез ОМЛ и предсказать прогноз в каждом конкретном случае. E. Papaemmanuil и соавт. [35] опубликовали данные о том, что при ОМЛ с NPM1+ ключевым фактором влияния на прогноз являются дополнительные генетические мутации, а не хромосомные перестройки, т. е. эта группа больных может классифицироваться вне зависимости от результатов кариотипирования. В рекомендациях ELN-2017 выделяется группа больных с NPM1+/FLT3-ITD- или NPM1+/FLT3-ITD ${ }^{\text {low }}$, которая имеет благоприятный прогно3, и группа NPM1+/FLT3$\mathrm{ITD}^{\text {high }}$, которая имеет промежуточный прогноз [36]. Больных с NPM1-/FLT3-ITD ${ }^{\text {low }}$ относят в группу промежуточного прогноза, тогда как пациентов с NPM1-/ FLT3-ITD $^{\text {high }}$ - неблагоприятного [29, 37, 38]. В нашем исследовании мы наблюдали аналогичные закономерности: пациенты с одиночной мутацией NPM1+ имели значительно более длительные медианы ОВ и БРВ по сравнению с остальной группой независимо от варианта кариотипа. Однако прогноз у больных с NPM1+/FLT3-ITD ${ }^{\text {low }}$ и NPM1+/FLT3-ITD ${ }^{\text {high }}$ не отличался. В настоящее время применение комбинации химиотерапии и ингибиторов тирозинкиназы FLT3 позволит улучшить результаты терапии в группе больных с FLT3-ITD [39].

Что касается других маркеров, таких как мутации в генах DNMT3A, IDH1/2, то на данный момент они не включены в классификацию ELN-2017 из-за противоречивости результатов разных исследований об их влиянии на прогноз [36]. В нашем исследовании негативное влияние (тенденция) мутаций в гене DNMT3A прослеживалось при оценке медианы OВ пациентов - 12 (DNMT3A+) и 15 мес. (DNMT3A-) $(p=0,112)$, что соответствует данным литературы [24, $40,41]$. Наличие мутаций в гене IDH1 коррелировало с лучшей ОВ по сравнению с группой без мутаций ( $p=0,092)$, а наличие полиморфизма rs11554137 в гене IDH1 - наоборот, с ухудшением ОВ в группе больных с НК $(p=0,186)$. Несмотря на то что имеются данные как об отсутствии, так и о неблагоприятном влиянии мутаций в гене IDH1 на прогноз, исследователи предполагают, что решающую роль при распределении в группы риска играют изменения в кариотипе и наличие дополнительных мутаций [42-44].

Наряду с активным внедрением молекулярногенетических методов диагностики в рутинную клиническую практику на передний план также выходит разработка новых препаратов направленного действия на конкретные онкогенные белки и микроокружение у больных ОМЛ: ингибиторов протеинкиназ, эпигенетических модуляторов, митохондриальных ингибиторов и антител [45]. Как известно, мутации генов эпигенетической регуляции, таких как DNMT3A и $I D H 1 / 2$, обнаруживают в ранних предшественниках лейкозных клеток, т. е. они считаются одним из первых событий в развитии ОМЛ. Важной особенностью мутаций в гене DNMT3A является их стабильность на разных этапах заболевания, даже в период ремиссии; как следствие, они могут быть причиной резистентности к химиотерапии $[46,47]$. Таким образом, ингибирование этих генетических аномалий позволит влиять на «founding» клон (который coдержит мутацию, произошедшую на первом этапе заболевания, и который в дальнейшем инициирует образование субклонов с дополнительными мутациями), тогда как таргетное воздействие на мутации в рецепторных тирозинкиназах (FLT3), которые, как правило, встречаются на более поздней стадии болезни, приведет к эрадикации основной массы опухолевых субклонов.

\section{ЗАКЛЮЧЕНИЕ}

Описание за последнее время большого количества повреждений генов, характерных для больных ОМЛ, множественные драйверные мутации, встречающиеся у 1 пациента, сложная система их кооперативного воздействия на прогноз служат основанием для продолжения работы над геномной классификацией ОМЛ.

\section{КОНФЛИКТЫ ИНТЕРЕСОВ}

Авторы заявляют об отсутствии конфликтов интересов.

\section{ИСТОЧНИКИ ФИНАНСИРОВАНИЯ}

Исследование не имело спонсорской поддержки.

\section{ВКЛАД АВТОРОВ}

Концепция и дизайн: И.С. Мартынкевич, О.В. Блау, Е.В. Мотыко.

Сбор и обработка данных: Е.В. Мотыко, О.В. Блау.

Предоставление материалов исследования: все авторы.

Анализ и интерпретация данных: Е.В. Мотыко, О.В. Блау.

Подготовка рукописи: Е.В. Мотыко.

Окончательное одобрение рукописи: все авторы.

\section{ЛИTEPATYPA/REFERENCES}

1. Schlenk RF, Dohner H. Genomic applications in the clinic: use in treatment paradigm of acute myeloid leukemia. Hematol Am Soc Hematol Educ Program. 2013;2013(1):324-30. doi: 10.1182/asheducation-2013.1.324.

2. Sanders MA, Valk PJ. The evolving molecular genetic landscape in acute myeloid leukaemia. Curr Opin Hematol. 2013;20(2):79-85. doi: 10.1097/ $\mathrm{MOH} .0 \mathrm{~b} 013 \mathrm{e} 32835 \mathrm{~d} 821 \mathrm{c}$.

3. Preisler H, Davis RB, Kirshner J, et al. Comparison of three remission induction regimens and two postinduction strategies for the treatment of acute nonlymphocytic leukemia: a cancer and leukemic group B study. Blood. 1987;69(5):1441-9.

4. Wiernik PH, Banks PLC, Case DC, et al. Cytarabine plus idarubicin or daunorubicin as induction and consolidation therapy for previously untreated adult patients with acute myeloid leukemia. Blood. 1992;79(2):313-9.

5. Алгоритмы диагностики и протоколы лечения заболеваний системь крови. Под ред. В.Г. Савченко. М.: Практика, 2018. Т. 1. 1008 с. 
Savchenko VG, ed. Algoritmy diagnostiki i protokoly lecheniya zabolevanii sistemy krovi. (Diagnostic algorithms and treatment protocols for blood system diseases.) Moscow: Praktika Publ.; 2018. Vol. 1. 1008 p. (In Russ)]

6. Bennett JM, Catovsky D, Daniel MT, et al. Proposals for the classification of the acute leukaemias. French-American-British (FAB) co-operative group. $\mathrm{Br} J$ Haematol. 1976;33(4):451-8. doi: 10.1111/j.1365-2141.1976.tb03563.x.

7. Heim S, Mitelman F. Cancer Cytogenetics: chromosomal and molecular genetic aberrations of tumor cells. 4th ed. Wiley-Blackwell: 2015. pp. 632. doi: 10.1002/9781118795569.

8. Jordan CT. Unique molecular and cellular features of acute myelogenous leukemia stem cells. Leukemia. 2002;16(4):559-62. doi: 10.1038/sj.leu.2402446.

9. Ding L, Ley TJ, Larson DE, et al. Clonal evolution in relapsed acute myeloid leukaemia revealed by whole-genome sequencing. Nature. 2012;481(7382):50610. doi: $10.1038 /$ nature10738.

10. Gerlinger M, Rowan AJ, Horswell S, et al. Intratumor heterogeneity and branched evolution revealed by multiregion sequencing. $\mathrm{N}$ Engl $\mathrm{J}$ Med. 2012;366(10):883-92. doi: 10.1056/NEJMoa1113205.

11. Campbell PJ, Pleasance ED, Stephens PJ, et al. Subclonal phylogenetic structures in cancer revealed by ultra-deep sequencing. Proc Natl Acad Sci USA 2008;105(35):13081-6. doi: 10.1073/pnas.0801523105.

12. Kottaridis PD, Gale RE, Frew ME, et al. The presence of a FLT3 internal tandem duplication in patients with acute myeloid leukemia (AML) adds important prognostic information to cytogenetic risk group and response to the first cycle of chemotherapy: analysis of 854 patients from the United Kingdom Medical Research Council AML 10 and 12 trials. Blood. 2001;98(6):1752-9. doi: 10.1182/ blood.v98.6.1752

13. Santos FP, Jones D, Qiao W, et al. Prognostic value of FLT3 mutations among different cytogenetic subgroups in acute myeloid leukemia. Cancer. 2011;117(10):2145-55. doi: 10.1002/cncr.25670.

14. Sallman DA, Lancet JE. What are the most promising new agents in acute myeloid leukemia? Curr Opin Hematol. 2017;24(2):99-107. doi: 10.1097/ $\mathrm{MOH} .0000000000000319$.

15. Thiede C, Koch S, Creutzig E, et al. Prevalence and prognostic impact of NPM1 mutations in 1485 adult patients with acute myeloid leukemia (AML). Blood. 2006:107(10):4011-20. doi: 10.1182/blood-2005-08-3167.

16. Dohner K, Schlenk RF, Habdank M, et al. Mutant nucleophosmin (NPM1) predicts favorable prognosis in younger adults with acute myeloid leukemia and normal cytogenetics: interaction with other gene mutations. Blood. 2005;106(12):3740-6. doi: 10.1182/blood-2005-05-2164.

17. Тилова Л.Р., Савинкова А.В., Жидкова Е.М. и др. Молекулярно-генетические нарушения в патогенезе опухолей системы крови и соответствующие им изменения сигнальных систем клетки. Клиническая онкогематология. 2017;10(2):235-49. doi: 10.21320/2500-2139-2017-10-2-235-249.

[Tilova LR, Savinkova AV, Zhidkova EM, et al. Molecular Genetic Abnormalities in the Pathogenesis of Hematologic Malignancies and Corresponding Changes in Cell Signaling Systems. Clinical oncohematology. 2017;10(2):235-49. doi: 10.21320/2500-2139-2017-10-2-235-249. (In Russ)]

18. Emadi A, Faramand R, Carter-Cooper B, et al. Presence of isocitrate dehydrogenase mutations may predict acute myeloid leukemia. Am J Hematol. 2015;90(5):E77-9. doi: 10.1002/ajh.23965.

19. Patel JP, Gonen M, Figueroa ME, et al. Prognostic relevance of integrated genetic profiling in acute myeloid leukemia. N Engl J Med. 2012;366(12):1079-89. doi: 10.1056/NEJMoa1112304.

20. Renneville A, Boissel N, Nibourel $\mathrm{O}$, et al. Prognostic significance of DNA methyltransferase $3 \mathrm{~A}$ mutations in cytogenetically normal acute myeloid leukemia: a study by the Acute Leukemia French Association. Leukemia. 2012;26(6):1247-54. doi: 10.1038/leu.2011.382.

21. Marcucci G, Maharry K, Wu Y-Z, et al. IDH1 and IDH2 gene mutations identify novel molecular subsets within de novo cytogenetically normal acute myeloid leukemia: a Cancer and Leukemia Group B study. J Clin Oncol. 2010;28(14):2348-55. doi: 10.1200/JCO.2009.27.3730

22. Paschka P, Schlenk RF, Gaidzik VI, et al. IDH1 and IDH2 mutations are frequent genetic alterations in acute myeloid leukemia and confer adverse prognosis in cytogenetically normal acute myeloid leukemia with NPM1 mutation without FLT3 internal tandem duplication. J Clin Oncol. 2010;28(22):3636-43. doi: 10.1200/JCO.2010.28.3762

23. Abbas $\mathrm{S}$, Lugthart $\mathrm{S}$, Kavelaars FG, et al. Acquired mutations in the genes encoding IDH1 and IDH2 both are recurrent aberrations in acute myeloid leukemia: prevalence and prognostic value. Blood. 2010;116(12):2122-6. doi: 10.1182/ blood-2009-11-250878

24. Thol F, Damm F, Ludeking A, et al. Incidence and prognostic influence of DNMT3A mutations in acute myeloid leukemia. J Clin Oncol. 2011;29(21):2889-96. doi: 10.1200/JCO.2011.35.4894.

25. Ley TJ, Miller C, Ding L, Raphael BJ, et al. Genomic and epigenomic landscapes of adult de novo acute myeloid leukemia. N Engl J Med. 2013;368(22):2059-74. doi: 10.1056/NEJMoa1301689.
26. Kihara $\mathrm{R}$, Nagata $\mathrm{Y}$, Kiyoi $\mathrm{H}$, et al. Comprehensive analysis of genetic alterations and their prognostic impacts in adult acute myeloid leukemia patients. Leukemia. 2014;28(8):1586-95. doi: 10.1038/leu.2014.55.

27. Ravandi F, Kantarjian H, Faderl S, et al. Outcome of patients with FLT3mutated acute myeloid leukemia in first relapse. Leuk Res. 2010;34(6):752-6. doi: 10.1016/j.leukres.2009.10.001.

28. Frohling S, Schlenk RF, Breitruck J, et al. Prognostic significance of activating FLT3 mutations in younger adults (16 to 60 years) with acute myeloid leukemia and normal cytogenetics: A study of the AML study group UIm. Blood. 2002;100(13):4372-80. doi: 10.1182/blood-2002-05-1440.

29. Schlenk RF, Kayser $S$, Bullinger $L$, et al. Differential impact of allelic ratio and insertion site in FLT3-ITD-positive AML with respect to allogeneic transplantation. Blood. 2014;124(23):3441-9. doi: 10.1182/blood-2014-05-578070.

30. Kim Y, Lee GD, Park J, et al. Quantitative fragment analysis of FLT3-ITD efficiently identifying poor prognostic group with high mutant allele burden or long ITD length. Blood Cancer J. 2015;5(8):e336. doi: 10.1038/bcj.2015.61.

31. Linch DC, Hills RK, Burnett AK, et al. Impact of FLT3ITD mutant allele level on relapse risk in intermediate-risk acute myeloid leukemia. Blood. 2014;124(2):273-6. doi: 10.1182/blood-2014-02-554667.

32. Brunet $\mathrm{S}$, Labopin $\mathrm{M}$, Esteve J, et al. Impact of FLT3 internal tandem duplication on the outcome of related and unrelated hematopoietic transplantation for adult acute myeloid leukemia in first remission: a retrospective analysis. J Clin Oncol. 2012;30(7):735-41. doi: 10.1200/JCO.2011.36.9868.

33. DeZern AE, Sung A, Kim S, et al. Role of allogeneic transplantation for FLT3/ ITD acute myeloid leukemia: outcomes from 133 consecutive newly diagnosed patients from a single institution. Biol Blood Marrow Transplant. 2011;17(9):1404-9. doi: 10.1016/j.bbmt.2011.02.003.

34. Islam M, Mohamed Z, Assenov Y. Differential analysis of genetic, epigenetic, and cytogenetic abnormalities in AML. Int J Genom. 2017;2017:2913648. doi: 10.1155/2017/2913648.

35. Papaemmanuil E, Gerstung M, Bullinger L, et al. Genomic classification and prognosis in acute myeloid leukemia. N Engl J Med. 2016;375(9):900-1. doi: 10.1056/NEJMc1608739.

36. Dohner H, Estey E, Amadori S, et al. Diagnosis and Management of Acute Myeloid Leukemia in Adults: Recommendations from an International Expert Panel, on Behalf of the European LeukemiaNet. Blood. 2010;115(3):453-74. doi: 10.1182/blood-2009-07-235358.

37. Gale RE, Green C, Allen C, et al. The impact of FLT3 internal tandem duplication mutant level, number, size, and interaction with NPM1 mutations in a large cohort of young adult patients with acute myeloid leukemia. Blood. 2008;111(5):2776-84. doi: 10.1182/blood-2007-08-109090.

38. Pratcorona $\mathrm{M}$, Brunet $\mathrm{S}$, Nomdedeu J, et al. Favorable outcome of patients with acute myeloid leukemia harboring a low-allelic burden FLT3-ITD mutation and concomitant NPM1 mutation: Relevance to post-remission therapy. Blood. 2013;121(14):2734-8. doi: 10.1182/blood-2012-06-431122.

39. Stone RM, Mandrekar S, Sanford BL, et al. The Multi-Kinase Inhibitor Midostaurin (M) Prolongs Survival Compared with Placebo $(P)$ in Combination with Daunorubicin (D)/Cytarabine (C) Induction (ind), High-Dose C Consolidation (consol), and As Maintenance (maint) Therapy in Newly Diagnosed Acute Myeloid Leukemia (AML) Patients (pts) Age 18-60 with FLT3 Mutations (muts): An International Prospective Randomized (rand) P-Controlled Double- Blind Trial (CALGB 10603/RATIFY [Alliance]). Blood. 2015;126(23): 6, abstract.

40. Ibrahem L, Mahfouz R, Elhelw L, et al. Prognostic significance of DNMT3A mutations in patients with acute myeloid leukemia. Blood Cells Mol Dis. 2015;54(1):84-9. doi: 10.1016/j.bcmd.2014.07.015

41. Ley $\mathrm{T}$, Ding $\mathrm{L}$, Walter $\mathrm{M}$, et al. DNMT3A mutations in acute myeloid leukemia. N Engl J Med. 2010;363(25):2424-33. doi: 10.1056/NEJMoa1005143.

42. Willander K, Falk I, Chaireti R, et al. Mutations in the isocitrate dehydrogenase 2 gene and IDH1 SNP 105C>T have a prognostic value in acute myeloid leukemia. Biomark Res. 2014;2(1):18. doi: 10.1186/2050-7771-2-18.

43. Xu Q, Li Y, Lv N, et al. Correlation between isocitrate dehydrogenase gene aberrations and prognosis of patients with acute myeloid leukemia: a systematic review and meta-analysis. Clin Cancer Res. 2017;23(15):4511-22. doi: 10.1158/1078 0432.CCR-16-2628.

44. Wagner K, Damm F, Gohring G, et al. Impact of IDH1 R132 mutations and an IDH1 single nucleotide polymorphism in cytogenetically normal acute myeloid leukemia: SNP rs11554137 is an adverse prognostic factor. J Clin Oncol. 2010;28(14):2356-64. doi: 10.1200/JCO.2009.27.6899.

45. Stein EM, Tallman MS. Emerging therapeutic drugs for AML. Blood. 2016;127(1):71-8. doi: 10.1182/blood-2015-07-604538.

46. Ploen GG, Nederby L, Guldberg P, et al. Persistence of DNMT3A mutations at long-term remission in adult patients with AML. Br J Haematol. 2014;167(4):47886. doi: $10.1111 /$ bjh.13062.

47. Gaidzik V, Weber D, Paschka P, et al. Monitoring of minimal residual disease (MRD) of DNMT3A mutations (DNMT3Amut) in acute myeloid leukemia (AML): a study of the AML Study Group (AMLSG). Blood. 2015;126(23):226, abstract. 